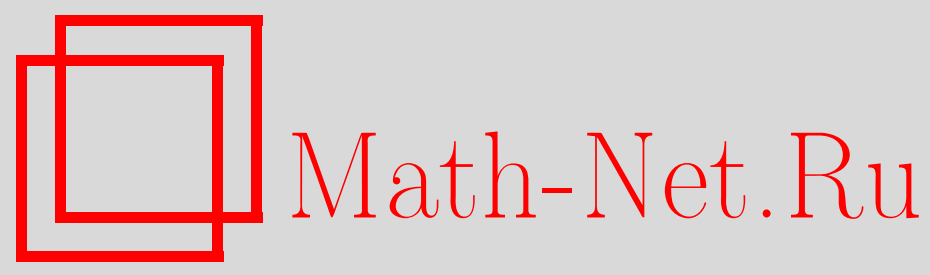

В. В. Коннов, Кокасательное расслоение проективного пространства и многообразие невырожденных нуль-пар, $M a-$ тем. заметки, 2001, том 70, выпуск 5, 718-735

DOI: https://doi.org/10.4213/mzm783

Использование Общероссийского математического портала Math-Net.Ru подразумевает, что вы прочитали и согласны с пользовательским соглашением http://www.mathnet.ru/rus/agreement

Параметры загрузки:

IP : 54.224 .60 .19

26 апреля 2023 г., $15: 19: 28$

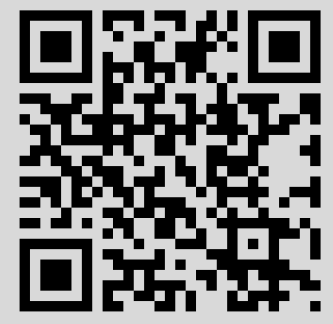




\title{
КОКАСАТЕЛЬНОЕ РАССЛОЕНИЕ ПРОЕКТИВНОГО ПРОСТРАНСТВА И МНОГООБРАЗИЕ НЕВЫРОЖДЕННЫХ НУЛЬ-ПАР
}

\section{В.В. Коннов}

\begin{abstract}
Невырожденная нуль-пара вещественного проективного пространства $P^{n}$ состоит из точки и неинцидентной ей гиперплоскости. Многообразие всех невырожденных нуль-пар $\mathfrak{N}$ обладает естественной келеровой структурой гиперболического типа постоянной ненулевой голоморфной секционной кривизны. В частности, $\mathfrak{N}$ - симплектическое многообразие. Как доказано в работе, многообразие $\mathfrak{N}$ несет структуру локально тривиального расслоения над проективным пространством $P^{n}$, типовым слоем которого является аффинное пространство. При этом ассоциированное векторное пространство каждого слоя естественно изоморфно кокасательному пространству к $P^{n}$. В работе найдено глобальное сечение этого расслоения, что позволило построить диффеоморфизм $\sigma$ между многообразием невырожденных нуль-пар и кокасательным расслоением проективного пространства. Основным результатом работы является доказательство теоремы о том, что построенный в явном виде диффеоморфизм $\sigma: \mathfrak{N} \rightarrow$ $T^{*} P^{n}$ является симплектоморфизмом относительно естественной симплектической структуры на $\mathfrak{N}$ и канонической симплектической структуры на $T^{*} P^{n}$.

Библиограффия: 6 названий.
\end{abstract}

1. Проективное пространство, его касательное и кокасательное расслоения. Пусть $P^{n}=P(W)$ - вещественное проективное пространство размерности $n$, порожденное векторным пространтвом $W$. Будем считать, что в пространстве $W$ фиксирован базис $\left\{e_{0}, e_{1}, \ldots, e_{n}\right\}$, относительно которого $\left(x^{0}, x^{1}, \ldots, x^{n}\right)$ - координатына $W$, a $\left(x^{0}: x^{1}: \cdots: x^{n}\right)$ - однородные координаты на $P(W)$. Структура $n$-мерного вещественно-аналитического многообразия определяется на $P(W)$ при помощи атласа $\mathscr{A}=$ $\left\{\left(A_{p}, f_{p}\right) \mid p=0,1, \ldots, n\right\}$, состоящего из аффинных карт. Здесь

$$
A_{p}=\left\{\left(x^{0}: x^{1}: \cdots: x^{n}\right) \in P(W) \mid x^{p} \neq 0\right\}
$$

- область карты,

$$
f_{p}:\left(x^{0}: x^{1}: \cdots: x^{n}\right) \rightarrow\left(y^{0}, \ldots, y^{p-1}, y^{p+1}, \ldots, y^{n}\right)=\left(\frac{x^{0}}{x^{p}}, \ldots, \frac{x^{p-1}}{x^{p}}, \frac{x^{p+1}}{x^{p}}, \ldots, \frac{x^{n}}{x^{p}}\right)
$$

- картирующее отображение.

Каноническая проекция р: $W \backslash 0 \rightarrow P(W)$ является аналитическим отображением и назьвается отображсением проективизации. Если вектор $a \in W$ порождает точку 
$A \in P(W)$, т.е. $\mathrm{p}(a)=A$, то точку $A$ будем отождествлять с одномерньм векторньм подпространством $\langle a\rangle$, натянутьм на вектор $a$. Итак, $A=\mathrm{p}(a)=\langle a\rangle$.

Рассмотрим короткую последовательность векторных пространств и их гомоморфизMOB

$$
\mathscr{O} \longrightarrow\langle a\rangle \stackrel{i}{\longrightarrow} T_{a} W \equiv W \stackrel{\left(\mathrm{p}_{*}\right) a}{\longrightarrow} T_{A} P(W) \longrightarrow \mathscr{O},
$$

где $i$ - гомоморфизм включения. Легко показать, что рассматриваемая последовательность является точной, т.е. $\operatorname{Im} i=\operatorname{ker}\left(\mathrm{p}_{*}\right)_{a}=\langle a\rangle$. Следовательно, каждьй вектор $a$, лежащий над точкой $A=\mathrm{p}(a)$, определяет естественньй изоморфизм

$$
\varphi_{a}: T_{A} P(W) \rightarrow W /\langle a\rangle,
$$

для которого $\varphi_{a} \circ\left(\mathrm{p}_{*}\right)_{a}=\pi$, где $\pi: W \rightarrow W /\langle a\rangle-$ каноническая проекция. В карте $A_{p}$ имеют место формулы

$$
\begin{gathered}
\left(\mathrm{p}_{*}\right)_{a}: b^{u} e_{u} \equiv b^{u} \frac{\partial}{\partial x^{u}} \mapsto\left(\mathrm{p}_{*}\right)_{a}\left(b^{u} \frac{\partial}{\partial x^{u}}\right)=\frac{1}{a^{p}}\left(b^{i_{p}}-\frac{a^{i_{p}}}{a^{p}} b^{p}\right) \frac{\partial}{\partial y^{i_{p}}} \\
\pi: b^{u} e_{u} \mapsto\langle a\rangle+b^{u} e_{u} \equiv\langle a\rangle+\left(b^{i_{p}}-b^{p} \frac{a^{i_{p}}}{a_{p}}\right) e_{i_{p}} \\
\varphi_{a}: c^{i_{p}} \frac{\partial}{\partial y^{i_{p}}} \mapsto\langle a\rangle+a^{p} c^{i_{p}} e_{i_{p}}, \quad\left(\varphi_{a}\right)^{-1}:\langle a\rangle+b^{i_{p}} e_{i_{p}} \mapsto \frac{1}{a^{p}} b^{i_{p}} \frac{\partial}{\partial y^{i_{p}}} .
\end{gathered}
$$

Здесь $p$ - фиксированный индекс из множества $\{0,1, \ldots, n\}$, по которому суммирование не производится. Суммирование производится по индексу $u=0,1, \ldots, n$, нумерующему глобальные координаты на $W$, и по индексу $i_{p}=0,1, \ldots, p-1, p+1, \ldots, n$, которьй нумерует локальные координаты в карте $A_{p}$.

Таким образом, имеем однопараметрический пучок изоморфизмов

$$
\varphi_{a}: T_{A} P(W) \rightarrow W /\langle a\rangle
$$

связанных соотношениями

$$
\varphi_{\lambda a}=\lambda \varphi_{a}, \quad\left(\varphi_{\lambda a}\right)^{-1}=\frac{1}{\lambda}\left(\varphi_{a}\right)^{-1} .
$$

Кокасательное пространство $T_{A}^{*} P(W)$ к проективному пространству $P(W)$ в точке $A=\mathrm{p}(a)$ будем отождествлять с подпространством $\operatorname{Ann}\langle a\rangle \subset W^{*}$, т.е. $T_{A}^{*} P(W)$ будем рассматривать как совокупность линейных форм на $W$, равных нулю на векторе $a$. Такой подход корректен, так как для любого вектора $a$, лежащего над точкой $\mathrm{p}(a)$, отображение

$$
\mathrm{p}_{a}^{*}=\pi^{*} \circ\left(\varphi_{a}^{-1}\right)^{*}: T_{A}^{*} P(W) \rightarrow \operatorname{Ann}\langle a\rangle \subset W^{*} \equiv T_{a}^{*} W
$$

является изоморфизмом на образ. Изоморфизм (на образ) $\pi^{*}=\mathrm{p}_{a}^{*} \circ \varphi_{a}^{*}$ позволяет отождествить пространство Ann $\langle a\rangle$ с пространством $(W /\langle a\rangle)^{*}$, сопряженньм для $W /\langle a\rangle$.

В карте $A_{p}$ имеют место формулы

$$
\begin{gathered}
\mathrm{p}_{a}^{*}: c_{i_{p}} d y^{i_{p}} \mapsto \mathrm{p}_{a}^{*}\left(c_{i_{p}} d y^{i_{p}}\right)=\frac{1}{a^{p}} c_{i_{p}}\left(d x^{i_{p}}-\frac{a^{i_{p}}}{a^{p}} d x^{p}\right)=\frac{1}{a^{p}} c_{i_{p}}\left(e^{i_{p}}-\frac{a^{i_{p}}}{a^{p}} e^{p}\right) \\
\left(\mathrm{p}_{a}^{*}\right)^{-1}: b_{i_{p}}\left(e^{i_{p}}-\frac{a^{i_{p}}}{a^{p}} e^{p}\right) \mapsto a^{p} b_{i_{p}} d y^{i_{p}}
\end{gathered}
$$


Заметим, что если $a^{p} \neq 0$, то любая форма из Ann $\langle a\rangle$ может быть записана в виде $b_{i_{p}}\left(e^{i_{p}}-a^{i_{p}} e^{p} / a^{p}\right)$.

Однопараметрический пучок изоморфизмов $\mathrm{p}_{a}^{*}$ обладает следующим свойством:

$$
\mathrm{p}_{\lambda a}^{*}=\frac{1}{\lambda} \mathrm{p}_{a}^{*}, \quad\left(\mathrm{p}_{\lambda a}^{*}\right)^{-1}=\lambda\left(\mathrm{p}_{\lambda a}^{*}\right)^{-1}
$$

Итак, проективное пространство $P(W)$, его касательное и кокасательное расслоения будем интерпретировать в терминах векторного пространства $W$ :

$$
P(W)=\bigcup_{a \in W \backslash 0}\langle a\rangle, \quad T P(W)=\bigcup_{a \in W \backslash 0} W /\langle a\rangle, \quad T^{*} P(W)=\bigcup_{a \in W \backslash 0} \operatorname{Ann}\langle a\rangle .
$$

Пусть теперь $W^{*}$ - векторное пространство, сопряженное для $W$. Тогда $P\left(W^{*}\right)-$ проективное пространство, двойственное для $P(W)$, т.е. пространство гиперплоскостей в $P(W)$. Будем считать, что базис $\left\{e^{0}, e^{1}, \ldots, e^{n}\right\}$ в $W^{*}$ является сопряженным для $\left\{e_{0}, e_{1}, \ldots, e_{n}\right\}$. Относительно этого базиса $\left(x_{0}, x_{1}, \ldots, x_{n}\right)$ - координаты на $W^{*}$, a $\left(x_{0}: x_{1}: \cdots: x_{n}\right)$ - однородные координаты на $P\left(W^{*}\right)$. Пусть $\mathscr{A}^{*}=\left\{\left(A^{p}, f^{p}\right) \mid p=0\right.$, $1, \ldots, n\}$ - атлас, состоящий из аффинных карт на $P\left(W^{*}\right)$. Здесь

$$
A^{p}=\left\{\left(x_{0}: x_{1}: \cdots: x_{n}\right) \in P\left(W^{*}\right) \mid x_{p} \neq 0\right\}
$$

- область карты,

$f^{p}:\left(x_{0}: x_{1}: \cdots: x_{n}\right) \rightarrow\left(y_{0}, \ldots, y_{p-1}, y_{p+1}, \ldots, y_{n}\right)=\left(\frac{x_{0}}{x_{p}}, \ldots, \frac{x_{p-1}}{x_{p}}, \frac{x_{p+1}}{x_{p}}, \ldots, \frac{x_{n}}{x_{p}}\right)$

- картирующее отображение.

Отображение проективизации из $W^{*} \backslash 0$ на $P\left(W^{*}\right)$ будем снова обозначать через $\mathrm{p}$. Если $\xi \in W^{*}$, то будем использовать отождествления $\Xi=\mathrm{p}(\xi)=\langle\xi\rangle, T_{\Xi} P\left(W^{*}\right) \cong$ $W^{*} /\langle\xi\rangle$ и $T_{\Xi}^{*} P\left(W^{*}\right) \cong \operatorname{Ann}\langle\xi\rangle$. Таким образом,

$$
P\left(W^{*}\right)=\bigcup_{\xi \in W^{*} \backslash 0}\langle\xi\rangle, \quad T P\left(W^{*}\right)=\bigcup_{\xi \in W^{*} \backslash 0} W^{*} /\langle\xi\rangle, \quad T^{*} P\left(W^{*}\right)=\bigcup_{\xi \in W^{*} \backslash 0} \operatorname{Ann}\langle\xi\rangle .
$$

\section{2. Дифференциальная геометрия многообразия невырожденных нуль-} пар.

ОПРеДЕЛЕниЕ. Нуль-парой в $P(W)$ называется [1] пара $(A, \Xi)$, где $A \in P(W)$ и $\Xi \in P\left(W^{*}\right)$. Нуль-пара называется вырожденной, если точка $A=\mathrm{p}(a)$ и гиперплоскость $\Xi=\mathrm{p}(\xi)$ инцидентны, т.е. $A \in \Xi$ (или, что эквивалентно, $\xi(a)=0$ ). В противном случае нуль-пара называется невырожденной.

Обозначим через $\mathfrak{M}$ множество всех нуль-пар. Ясно, что $\mathfrak{M}=P(W) \times P\left(W^{*}\right)$ является $2 n$-мерным вещественно-аналитическим многообразием относительно структуры прямого произведения аналитических многообразий $P(W)$ и $P\left(W^{*}\right)$. Естественньй атлас на $\mathfrak{M}$ имеет вид

$$
\mathscr{A} \times \mathscr{A}^{*}=\left\{\left(A_{p} \times A^{q}, f_{p} \times f^{q}\right) \mid p, q=0,1, \ldots, n\right\} .
$$


Многообразие $\mathfrak{M}$ связно и компактно.

Пусть $\mathfrak{M}_{0}$ - подмножество в $\mathfrak{M}$, состоящее из всех вырожденных нуль-пар. Если $a=a^{u} e_{u}-$ ненулевой вектор, то $\left(a^{0}: a^{1}: \cdots: a^{n}\right)$ - однородные координаты точки $A=\mathrm{p}(a) \in P(W)$. Если же $\xi=\xi_{u} e^{u}-$ ненулевой ковектор, то $\left(\xi_{0}: \xi_{1}: \cdots: \xi_{n}\right)$ - однородные координаты гиперплоскости $\Xi=\mathrm{p}(\xi) \in P\left(W^{*}\right)$ (или, что тоже самое, ее тангенииальные координаты). Упорядоченный набор из $2 n+2$ чисел $\left(a^{0}: a^{1}: \cdots: a^{n}\right.$; $\left.\xi_{0}: \xi_{1}: \cdots: \xi_{n}\right)$ называется однородными координатами нуль-пары $(A, \Xi)$. В однородных координатах условие вырожденности нуль-пары $(A, \Xi)$ имеет вид

$$
a^{0} \xi_{0}+a^{1} \xi_{1}+\cdots+a^{n} \xi_{n}=0
$$

Это уравнение определяет $\mathfrak{M}_{0}$ как гладкую гиперповерхность в $\mathfrak{M}$. Ясно, что $\mathfrak{M}_{0}$ замкнуто в $\mathfrak{M}$.

Пусть $\mathfrak{N}=\mathfrak{M} \backslash \mathfrak{M}_{0}-$ совокупность всех невырожденных нуль-пар. Ясно, что $\mathfrak{N}-$ открытое подмногообразие в $\mathfrak{M}$. Естественньй атлас на $\mathfrak{N}$ имеет вид

$$
\left.\left(\mathscr{A} \times \mathscr{A}^{*}\right)\right|_{\mathfrak{N}}=\left\{\left(\left(A_{p} \times A^{q}\right) \cap \mathfrak{N},\left.\left(f_{p} \times f^{q}\right)\right|_{\mathfrak{N}}\right) \mid p, q=0,1, \ldots, n\right\} .
$$

Важно отметить, что любая невырожденная нуль-пара $(\mathrm{p}(a), \mathrm{p}(\xi))$ принадлежит одной из областей $\left(A_{p} \times A^{q}\right) \cap \mathfrak{N}$ атласа $\left.\left(\mathscr{A} \times \mathscr{A}^{*}\right)\right|_{\mathfrak{N}}$ с одинаковьми номерами $p$ и $q$. Действительно, условие невырожденности $\xi(a) \neq 0$ означает, что хотя бы одно из слагаемых в сумме $\xi_{0} a^{0}+\xi_{1} a^{1}+\cdots+\xi_{n} a^{n}$ отлично от нуля. Пусть не равно нулю слагаемое $\xi_{p} a^{p}$. Тогда $(\mathrm{p}(a), \mathrm{p}(\xi)) \in\left(A_{p} \times A^{p}\right) \cap \mathfrak{N}$. Следовательно, $\left\{\left(A_{p} \times A^{p}\right) \cap \mathfrak{N}\right\}_{p=0,1, \ldots, n}-$ открытое покрытие на $\mathfrak{N}$. Атлас

$$
\operatorname{diag}\left(\left.\left(\mathscr{A} \times \mathscr{A}^{*}\right)\right|_{\mathfrak{N}}\right) \equiv\left\{\left(\left(A_{p} \times A^{p}\right) \cap \mathfrak{N}, f_{p} \times f^{p}\right) \mid p=0,1, \ldots, n\right\}
$$

назовем каноническим аффинным атласом на $\mathfrak{N}$.

Так как на многообразии $\mathfrak{N}$ определена структура почти произведения, то

$$
T_{(\langle a\rangle,\langle\xi\rangle)} \mathfrak{N}=T_{\langle a\rangle} P(W) \oplus T_{\langle\xi\rangle} P\left(W^{*}\right) \cong(W /\langle a\rangle) \oplus\left(W^{*} /\langle\xi\rangle\right) .
$$

Канонический инволютивньй автоморфизм I, связанньй со структурой прямого произведения, в области карты $\left(A_{p} \times A^{p}\right) \cap \mathfrak{N}$ задается так:

$$
\mathbf{I}: c_{i_{p}} \frac{\partial}{\partial y^{i_{p}}} \oplus c^{i_{p}} \frac{\partial}{\partial y_{i_{p}}} \mapsto-c_{i_{p}} \frac{\partial}{\partial y^{i_{p}}} \oplus c^{i_{p}} \frac{\partial}{\partial y_{i_{p}}} .
$$

ПРЕДЛОЖЕНИЕ 1. Многообразие невырожденных нуль-пар $\mathfrak{N}$ является симплектическим многообразием.

ДОКАЗАТЕЛЬСТВО. Пусть $u, v=0,1, \ldots, n$, а $p$ - фиксированньй индекс из множества $\{0,1, \ldots, n\}$, по которому суммирование не производится. Кроме того, положим

$$
i_{p}, j_{p}, k_{p}=0,1, \ldots, p-1, p+1, \ldots, n \text {. }
$$

Для каждого $p \in\{0,1, \ldots, n\}$ определим симплектическую 2-форму в области

$$
\left(f_{p} \times f^{p}\right)\left(\left(A_{p} \times A^{p}\right) \cap \mathfrak{N}\right) \subset \mathbb{R}^{2 n}
$$


канонического атласа на $\mathfrak{N}$ по формуле

$$
\underset{p}{\Omega}=\frac{1}{\left(1+y_{k_{p}} y^{k_{p}}\right)^{2}}\left(\left(1+y_{k_{p}} y^{k_{p}}\right) \delta_{j_{p}}^{i_{p}}-y^{i_{p}} y_{j_{p}}\right) d y_{i_{p}} \wedge d y^{j_{p}}
$$

где $y^{i_{p}}=x^{i_{p}} / x^{p}$ и $y_{i_{p}}=x_{i_{p}} / x_{p}$ - локальные координаты на $\left(A_{p} \times A^{p}\right) \cap \mathfrak{N}$.

1. Докажем, что 2-форма $\left(f_{p} \times f^{p}\right)^{*} \Omega_{p}$ не зависит от выбора карты, т.е.

$$
\left(f_{p} \times f^{p}\right)^{*} \underset{p}{\Omega_{x}}=\left(f_{q} \times f^{q}\right)^{*} \Omega_{q} \quad \forall x \in\left(A_{p} \times A^{p}\right) \cap\left(A_{q} \times A^{q}\right) \cap \mathfrak{N} .
$$

Введем обозначения $\Delta=\left\{(a, \xi) \in W \times W^{*} \mid \xi(a)=0\right\}, \mathscr{W}=\left(W \times W^{*}\right) \backslash \Delta$ и рассмотрим регулярное сюрьективное отображение $\Pi: \mathscr{W} \rightarrow \mathfrak{N}$, определяемое следующим образом

$$
\Pi:(a, \xi) \mapsto(\mathrm{p}(a), \mathrm{p}(\xi)) .
$$

Тогда для любой пары $(a, \xi) \in \mathscr{W}$ отображение антиувлечения

$$
\Pi_{(a, \xi)}^{*}: \Lambda_{2} \mathfrak{N}_{\Pi(a, \xi)} \rightarrow \Lambda_{2} \mathscr{W}_{(a, \xi)} \equiv\left(W^{*} \oplus W\right) \wedge\left(W^{*} \oplus W\right)
$$

является линейньм отображением, для которого $\operatorname{ker} \Pi_{(a, \xi)}^{*}=0$. Найдем форму $\Pi_{(a, \xi)}^{*}$ 。 $\left(f_{p} \times f^{p}\right)^{*} \underset{p}{\Omega}$, увлеченную из $\left(f_{p} \times f^{p}\right)\left(\left(A_{p} \times A^{p}\right) \cap \mathfrak{N}\right) \subset \mathbb{R}^{2 n}$ на $\mathscr{W}$. Имеем

$$
\begin{aligned}
& \Pi_{(a, \xi)}^{*} \circ\left(f_{p} \times f^{p}\right){\underset{p}{\Omega}}_{\Omega}=\Pi_{(a, \xi)}^{*}\left(\frac{1}{\left(1+y_{k_{p}} y^{k_{p}}\right)^{2}}\left(\left(1+y_{k_{p}} y^{k_{p}}\right) \delta_{j_{p}}^{i_{p}}-y^{i_{p}} y_{j_{p}}\right) d y_{i_{p}} \wedge d y^{j_{p}}\right) \\
& =\frac{a^{p} \xi_{p}}{\xi(a)^{2}}\left(\delta_{j_{p}}^{i_{p}} \xi(a)-a^{i_{p}} \xi_{j_{p}}\right) d\left(\frac{x_{i_{p}}}{x_{p}}\right)_{\xi} \wedge d\left(\frac{x^{j_{p}}}{x^{p}}\right)_{a} \\
& =\frac{a^{p} \xi_{p}}{a^{p} a^{p} \xi_{p} \xi_{p} \xi(a)^{2}}\left(\delta_{j_{p}}^{i_{p}} \xi(a)-a^{i_{p}} \xi_{j_{p}}\right)\left(\xi_{p} d x_{i_{p}}-\xi_{i_{p}} d x_{p}\right) \wedge\left(a^{p} d x^{j_{p}}-a^{j_{p}} d x^{p}\right) \\
& =\frac{1}{\xi(a)^{2}}\left(\delta_{j_{p}}^{i_{p}} \xi(a)-a^{i_{p}} \xi_{j_{p}}\right) d x_{i_{p}} \wedge d x^{j_{p}}-\frac{1}{a^{p} \xi(a)^{2}}\left(\xi(a) a^{i_{p}}-a^{i_{p}} \xi_{j_{p}} a^{j_{p}}\right) d x_{i_{p}} \wedge d x^{p} \\
& -\frac{1}{\xi_{p} \xi(a)^{2}}\left(\xi(a) \xi_{j_{p}}-a^{i_{p}} \xi_{i_{p}} \xi_{j_{p}}\right) d x_{p} \wedge d x^{j_{p}} \\
& +\frac{1}{a^{p} \xi_{p} \xi(a)^{2}}\left(\xi(a) \xi_{j_{p}} a^{j_{p}}-a^{i_{p}} \xi_{i_{p}} \xi_{j_{p}} a^{j_{p}}\right) d x_{p} \wedge d x^{p} \\
& =\frac{1}{\xi(a)^{2}}\left(\delta_{j_{p}}^{i_{p}} \xi(a)-a^{i_{p}} \xi_{j_{p}}\right) d x_{i_{p}} \wedge d x^{j_{p}}-\frac{1}{a^{p} \xi(a)^{2}}\left(\xi_{p} a^{p} a^{i_{p}}\right) d x_{i_{p}} \wedge d x^{p} \\
& -\frac{1}{\xi_{p} \xi(a)^{2}}\left(\xi_{p} a^{p} \xi_{j_{p}}\right) d x_{p} \wedge d x^{j_{p}}+\frac{1}{a^{p} \xi_{p} \xi(a)^{2}}\left(\xi_{p} a^{p} \xi_{j_{p}} a^{j_{p}}\right) d x_{p} \wedge d x^{p} \\
& =\frac{1}{\xi(a)^{2}}\left(\delta_{j_{p}}^{i_{p}} \xi(a)-a^{i_{p}} \xi_{j_{p}}\right) d x_{i_{p}} \wedge d x^{j_{p}}-\frac{1}{\xi(a)^{2}}\left(\xi_{p} a^{i_{p}}\right) d x_{i_{p}} \wedge d x^{p} \\
& -\frac{1}{\xi(a)^{2}}\left(a^{p} \xi_{j_{p}}\right) d x_{p} \wedge d x^{j_{p}}+\frac{1}{\xi(a)^{2}}\left(\xi_{j_{p}} a^{j_{p}}\right) d x_{p} \wedge d x^{p} \\
& =\frac{1}{\xi(a)^{2}}\left(\delta_{j_{p}}^{i_{p}} \xi(a)-a^{i_{p}} \xi_{j_{p}}\right) d x_{i_{p}} \wedge d x^{j_{p}}+\frac{1}{\xi(a)^{2}}\left(\delta_{p}^{i_{p}} \xi(a)-\xi_{p} a^{i_{p}}\right) d x_{i_{p}} \wedge d x^{p}
\end{aligned}
$$




$$
\begin{aligned}
& +\frac{1}{\xi(a)^{2}}\left(\delta_{j_{p}}^{p} \xi(a)-a^{p} \xi_{j_{p}}\right) d x_{p} \wedge d x^{j_{p}}+\frac{1}{\xi(a)^{2}}\left(\delta_{p}^{p} \xi(a)-\xi_{p} a^{p}\right) d x_{p} \wedge d x^{p} \\
= & \frac{1}{\xi(a)^{2}} d x_{i_{p}} \wedge\left(\left(\delta_{j_{p}}^{i_{p}} \xi(a)-a^{i_{p}} \xi_{j_{p}}\right) d x^{j_{p}}-\left(\delta_{p}^{i_{p}} \xi(a)-a^{i_{p}} \xi_{p} a^{p}\right) d x^{p}\right) \\
& +\frac{1}{\xi(a)^{2}} d x_{p} \wedge\left(\left(\delta_{j_{p}}^{p} \xi(a)-\xi_{j_{p}} a^{p}\right) d x^{j_{p}}-\left(\xi(a) \delta_{p}^{p}-a^{p} \xi_{p}\right) d x^{p}\right) \\
= & \frac{1}{\xi(a)^{2}}\left(d x_{i_{p}} \wedge\left(\delta_{u}^{i_{p}} \xi(a)-a^{i_{p}} \xi_{u}\right) d x^{u}+d x_{p} \wedge\left(\delta_{u}^{p} \xi(a)-a^{p} \xi_{u}\right) d x^{u}\right) \\
= & \frac{1}{\xi(a)^{2}} d x_{v} \wedge\left(\delta_{u}^{v} \xi(a)-a^{v} \xi_{u}\right) d x^{u}=\frac{1}{\xi(a)^{2}}\left(\delta_{u}^{v} \xi(a)-a^{v} \xi_{u}\right) d x_{v} \wedge d x^{u} .
\end{aligned}
$$

Итак,

$$
\Pi_{(a, \xi)}^{*} \circ\left(f_{p} \times f^{p}\right)^{*} \underset{p}{\Omega}=\frac{1}{\xi(a)^{2}}\left(\delta_{u}^{v} \xi(a)-a^{v} \xi_{u}\right) d x_{v} \wedge d x^{u} .
$$

Если $q$ - другой индекс из множества $\{0,1, \ldots, n\}$, то

$$
\Pi_{(a, \xi)}^{*} \circ\left(f_{p} \times f^{p}\right)^{*} \underset{p}{\Omega}=\Pi_{(a, \xi)}^{*} \circ\left(f_{q} \times f^{q}\right)^{*} \underset{q}{\Omega},
$$

т.e.

$$
\Pi_{(a, \xi)}^{*}\left(\left(f_{p} \times f^{p}\right)^{*} \underset{p}{\Omega}-\left(f_{q} \times f^{q}\right)^{*} \underset{q}{\Omega}\right)=0 \quad \forall(a, \xi) \in \Pi^{-1}\left(\left(A_{p} \times A^{p}\right) \cap\left(A_{q} \times A^{q}\right) \cap \mathfrak{N}\right) .
$$

В силу того, что $\operatorname{ker} \Pi_{(a, \xi)}^{*}=0 \quad \forall(a, \xi) \in \mathscr{W}$, приходим к вьводу, что

$$
\left(f_{p} \times f^{p}\right)^{*} \underset{p}{\Omega_{x}}=\left(f_{q} \times f^{q}\right)^{*} \underset{q}{\Omega_{x}} \quad \forall x \in\left(A_{p} \times A^{p}\right) \cap\left(A_{q} \times A^{q}\right) \cap \mathfrak{N} .
$$

Следовательно, существует единственная 2-форма $\Omega$, глобально определенная на $\mathfrak{N}$ при помоши условия $\left.\Omega\right|_{\left(A_{p} \times A^{p}\right) \cap \mathfrak{N}}=\left(f_{p} \times f^{p}\right) * \underset{p}{\Omega}$. При этом

$$
\Pi^{*} \Omega=\frac{1}{\left(x_{w} x^{w}\right)^{2}}\left(\left(x_{w} x^{w}\right) \delta_{u}^{v}-x^{v} x_{u}\right) d x_{v} \wedge d x^{u} .
$$

ЗАмЕЧАНИЕ 1. Последней формуле удобно придать векторую интерпретацию, отождествив форму $\Omega_{(\langle a\rangle,\langle\xi\rangle)}$ с формой $\xi(a) \cdot \Pi_{(a, \xi)}^{*} \Omega$. Тогда из $(3)$ находим, что для любых векторов $b, c \in W$ и ковекторов $\eta, \zeta \in W^{*}$ справедлива формула

$$
\Omega_{(\langle a\rangle,\langle\xi\rangle)}(b \oplus \eta ; c \oplus \zeta)=\frac{1}{\xi(a)}\left(\left|\begin{array}{cc}
\xi(a) & \xi(c) \\
\eta(a) & \eta(c)
\end{array}\right|-\left|\begin{array}{cc}
\xi(a) & \xi(b) \\
\zeta(a) & \zeta(b)
\end{array}\right|\right) .
$$

Заметим, что эта формула инвариантна относительно преобразований вида

$$
b \mapsto b+\langle a\rangle, \quad \eta \mapsto \eta+\langle\xi\rangle, \quad c \mapsto c+\langle a\rangle, \quad \zeta \mapsto \zeta+\langle\xi\rangle
$$

Кроме того, формула (3) инвариантна относительно преобразований

$$
a \mapsto \lambda a, \quad \xi \mapsto \mu \xi
$$


Именно для этого был использован нормирующий множитель $\xi(a)$.

2. Теперь докажем, что форма $\Omega$ невырождена. Достаточно проверить, что $n$-матрища $B=\left(\delta_{j_{p}}^{i_{p}}\left(y_{k_{p}} y^{k_{p}}+1\right)-y^{i_{p}} y_{j_{p}}\right)$ невырождена. Отметим, что в области карты $\left(A_{p} \times A^{p}\right) \cap \mathfrak{N}$ справедливо неравенство $y_{k_{p}} y^{k_{p}}+1 \neq 0$.

Если $y_{k_{p}}=0$ или $y^{k_{p}}=0$ для всех $k_{p}$, то утверждение очевидно. Поэтому предположим, что матрища $C=\left(y_{j_{p}} y^{i_{p}}\right)$ имеет ранг 1. Найдем характеристический многочлен матрицы $C$ :

$$
\operatorname{det}(\lambda E-C)=\lambda^{n}-c_{1} \lambda^{n-1}+c_{2} \lambda^{n-2}+\cdots+(-1)^{n} c_{n},
$$

где $c_{k}$ - сумма определителей $k$-го порядка матрицы $C$, стоящих на ее главной диагонали. В частности, $c_{1}=\operatorname{tr} C$, a $c_{n}=\operatorname{det} C$. Так как $\operatorname{rank} C=1$, то $c_{2}=c_{3}=\cdots=c_{n}=0$. Следовательно,

$$
\operatorname{det}(\lambda \cdot E-C)=\lambda^{n}-\operatorname{tr} C \cdot \lambda^{n-1}
$$

Отсюда

$$
\begin{aligned}
\operatorname{det} B & =\operatorname{det}\left(\delta_{j_{p}}^{i_{p}}\left(y_{k_{p}} y^{k_{p}}+1\right)-y_{j_{p}} y^{i_{p}}\right)=\operatorname{det}\left(\left(1+y_{k_{p}} y^{k_{p}}\right) E-C\right) \\
& =\left(1+y_{k_{p}} y^{k_{p}}\right)^{n}-\operatorname{tr} C\left(1+y_{k_{p}} y^{k_{p}}\right)^{n-1} \\
& =\left(1+y_{k_{p}} y^{k_{p}}\right)^{n}-\left(y_{j_{p}} y^{j_{p}}\right)\left(1+y_{k_{p}} y^{k_{p}}\right)^{n-1} \\
& =\left(1+y_{k_{p}} y^{k_{p}}\right)^{n-1}\left(1+y_{k_{p}} y^{k_{p}}-y_{j_{p}} y^{j_{p}}\right)=\left(1+y_{k_{p}} y^{k_{p}}\right)^{n-1} \neq 0 .
\end{aligned}
$$

3. Докажем, что почти симплектическая структура, определяемая формой $\Omega$, интегрируема. Для этого докажем, что форма $\Omega$ точна на $\mathfrak{N}$. Для каждого индекса $p \in\{0$, $1, \ldots, n\}$ в области $\left(f_{p} \times f^{p}\right)\left(\left(A_{p} \times A^{p}\right) \cap \mathfrak{N}\right) \subset \mathbb{R}^{2 n}$ канонического атласана $\mathfrak{N}$ определим 1-форму $\underset{p}{\omega}$ по формуле

$$
\underset{p}{\omega}=\sum_{i_{p}}\left(\frac{y_{i_{p}}}{1+y_{k_{p}} y^{k_{p}}}-\frac{y^{i_{p}}}{1+\sum y^{k_{p}} y^{k_{p}}}\right) d y^{i_{p}} .
$$

Легко проверяется, что

$$
\Pi_{(a, \xi)}^{*} \circ\left(f_{p} \times f^{p}\right)^{*} \underset{p}{\omega}=\sum_{u}\left(\frac{\xi_{u}}{\xi(a)}-\frac{a^{u}}{\sum a^{v} a^{v}}\right) d x^{u},
$$

т.е. форма $\Pi_{(a, \xi)}^{*} \circ\left(f_{p} \times f^{p}\right)^{*} \underset{p}{\omega}$ не зависит от выбора карты:

$$
\Pi_{(a, \xi)}^{*} \circ\left(f_{p} \times f^{p}\right)^{*} \underset{p}{\omega}=\Pi_{(a, \xi)}^{*} \circ\left(f_{q} \times f^{q}\right)^{*} \underset{q}{\omega} \quad \forall p, q \in\{0,1, \ldots, n\} .
$$

И так как $\operatorname{ker} \Pi_{(a, \xi)}^{*}=0$, то

$$
\left(f_{p} \times f^{p}\right)^{*}{ }_{p} \omega_{x}=\left(f_{q} \times f^{q}\right)^{*}{\underset{q}{\alpha}}_{x} \quad \forall x \in\left(A_{p} \times A^{p}\right) \cap\left(A_{q} \times A^{q}\right) \cap \mathfrak{N} .
$$

Следовательно, на $\mathfrak{N}$ существует единственная 1-форма $\omega$, удовлетворяющая условию

$$
\left.\omega\right|_{\left(A_{p} \times A^{p}\right) \cap \mathfrak{N}}=\left(f_{p} \times f^{p}\right)^{*} \underset{p}{\omega} .
$$

При этом

$$
\Pi^{*} \omega=\sum_{u}\left(\frac{x_{u}}{x_{w} x^{w}}-\frac{x^{u}}{\sum x^{w} x^{w}}\right) d x^{u} .
$$

Осталось заметить, что формы $\omega$ и $\Omega$ удовлетворяют тождеству $d \omega=\Omega$, которое непосредственно проверяется в каждой области $\left(A_{p} \times A^{p}\right) \cap \mathfrak{N}$.

Итак, многообразие невырожденных нуль-пар $\mathfrak{N}$ является симплектическим многообразием. 
ПРЕДЛОЖЕНИЕ 2. Многообразие невырожденных нуль-пар $\mathfrak{N}$ является почти эрмитовым многообразием гиперболического типа, при этом фундаментальная форма структуры совпадает с симплектической формой $\Omega$.

ДокАЗАТЕЛЬСТво. Для каждого индекса $p \in\{0,1, \ldots, n\}$ определим гиперболическую метрику в области $\left(f_{p} \times f^{p}\right)\left(\left(A_{p} \times A^{p}\right) \cap \mathfrak{N}\right) \subset \mathbb{R}^{2 n}$ канонического атласа на $\mathfrak{N}$ по формуле

$$
\underset{p}{\Psi}=\frac{1}{\left(1+y_{k_{p}} y^{k_{p}}\right)^{2}}\left(y^{i_{p}} y_{j_{p}}-\left(1+y_{k_{p}} y^{k_{p}}\right) \delta_{j_{p}}^{i_{p}}\right) d y_{i_{p}} \odot d y^{j_{p}} .
$$

Символ ‘ $\odot$ ' в формуле (5) и ниже обозначает симметрическое тензорное произведение; например,

$$
\left(\theta_{1} \odot \theta_{2}\right)(\xi, \eta)=\frac{1}{2}\left(\theta_{1}(\xi) \theta_{2}(\eta)+\theta_{2}(\xi) \theta_{1}(\eta)\right) .
$$

Форма $\underset{p}{\Psi}$ невырождена (доказательство аналогично п. 2 из предложения 1 ) и имеет сигнатуру $(n, n)$. Производя вычисления, аналогичные п. 1 из доказательства предложения 1 , найдем

$$
\Pi_{(a, \xi)}^{*} \circ\left(f_{p} \times f^{p}\right)^{*} \underset{p}{\Psi}=\frac{1}{\xi(a)^{2}}\left(a^{u} \xi_{v}-\xi(a) \delta_{v}^{u}\right) d x_{u} \odot d x^{v}
$$

т.е.

$$
\Pi_{(a, \xi)}^{*} \circ\left(f_{p} \times f^{p}\right)^{*} \underset{p}{\Psi}=\Pi_{(a, \xi)}^{*} \circ\left(f_{q} \times f^{q}\right)^{*} \underset{q}{\Psi} \quad \forall p, q \in\{0,1, \ldots, n\} .
$$

И так как $\operatorname{ker} \Pi_{(a, \xi)}^{*}=0$, то

$$
\left(f_{p} \times f^{p}\right)^{*} \underset{p}{\Psi_{x}}=\left(f_{q} \times f^{q}\right)^{*} \underset{q}{\Psi_{x}} \quad \forall x \in\left(A_{p} \times A^{p}\right) \cap\left(A_{q} \times A^{q}\right) \cap \mathfrak{N} .
$$

Следовательно, на $\mathfrak{N}$ существует единственная псевдориманова метрика $\Psi$, удовлетворяющая условию

$$
\left.\Psi\right|_{\left(A_{p} \times A^{p}\right) \cap \mathfrak{N}}=\left(f_{p} \times f^{p}\right)^{*} \underset{p}{\Psi}
$$

При этом

$$
\Pi^{*} \Psi=\frac{1}{\left(x_{w} x^{w}\right)^{2}}\left(x_{u} x^{v}-\left(x_{w} x^{w}\right) \delta_{v}^{u}\right) d x_{u} \odot d x^{v} .
$$

Осталось заметить, что формы $\Psi, \Omega$ и оператор почти произведения I связаны соотношениями

$$
\mathbf{I}^{2}=\mathrm{id}, \quad \Psi(\mathbf{I} X, \mathbf{I} Y)=-\Psi(X, Y), \quad \Omega(X, Y)=\Psi(X, \mathbf{I} Y) \quad \forall X, Y \in \mathfrak{X}(\mathfrak{N})
$$

Таким образом, пара $\{\Psi, \mathbf{I}\}$ является внутренним образом определенной почти-эрмитовой структурой гиперболического типа на многобразии $\mathfrak{N}$. При этом симплектическая форма $\Omega$ является фундаментальной 2-формой этой структуры. 
ЗАмЕчАниЕ 2. В векторной интерпритации (ср. с замечанием 1) эрмитову метрику $\Psi_{(\langle a\rangle,\langle\xi\rangle)}$ будем отождествлять с формой $\xi(a) \cdot \Pi_{(a, \xi)}^{*} \Psi$. Тогда из $(6)$ находим, что для любых векторов $b, c \in W$ и ковекторов $\eta, \zeta \in W^{*}$ справедлива формула

$$
\Psi_{(\langle a\rangle,\langle\xi\rangle)}(b \oplus \eta ; c \oplus \zeta)=-\frac{1}{\xi(a)}\left(\left|\begin{array}{cc}
\xi(a) & \xi(c) \\
\eta(a) & \eta(c)
\end{array}\right|+\left|\begin{array}{cc}
\xi(a) & \xi(b) \\
\zeta(a) & \zeta(b)
\end{array}\right|\right)
$$

Так же, как и (4), формула (7) инвариантна относительно преобразований вида

$$
\begin{aligned}
b \mapsto b+\langle a\rangle, \quad \eta \mapsto \eta+\langle\xi\rangle, & c \mapsto c+\langle a\rangle, \quad \zeta \mapsto \zeta+\langle\xi\rangle, \\
a \mapsto \lambda a, \quad \xi \mapsto \mu \xi & \mapsto \quad l
\end{aligned}
$$

Стандартные вычисления приводят к формулам $\nabla \Psi=\nabla \Omega=\nabla \mathbf{I}=0$, где $\nabla$ - связность Леви-Чивита метрики $\Psi$. Секционная кривизна в двумерном направлении $X \wedge Y$ вычисляется так:

$$
k(X \wedge Y)=\frac{1}{4}+\frac{3}{4} \cdot \frac{\Omega(X, Y)^{2}}{\Psi(X, Y)^{2}-\Psi(X, X) \Psi(Y, Y)} .
$$

Из последней формулы следует, в частности, что $k(X \wedge \mathbf{I} X)=1$. Таким образом, многообразие невырохденных нуль-пар $\mathfrak{N}$ является келеровым многообразием гиперболического типа постоянной ненулевой голоморфной секционной кривизны. Более того, любое келерово многообразие гиперболического типа постоянной ненулевой голоморфной секционной кривизны локально голоморфно изометрично многообразию невырожденных нуль-пар $\mathfrak{N}$ (см. [2]). Кроме того, несложнопоказать, что многообразие невырожденных нуль-пар несет структуру однородного пространства:

$$
\mathfrak{N}=\mathrm{GL}(n+1, \mathbb{R}) / \mathrm{GL}(1, \mathbb{R}) \times \mathrm{GL}(n, \mathbb{R}) .
$$

Таким образом, многообразие невырожденных нуль-пар $\mathfrak{N}$ является аналогом (неплоских) комплексных пространственных форм в гиперболической геометрии. При этом аналогом вполне вещественных подмногообразий на многообразии $\mathfrak{N}$ являются лагранжевы подмногообразия симплектической структуры $\Omega$. Прямым следствием формулы (8) является тот факт, что на лагранжевых подмногообразиях секционная кривизна метрики $\Psi$ постоянна и равна $1 / 4$.

Далее нас будет интересовать лишь естественная симплектическая структура на $\mathfrak{N}$, порожденная фундаментальной формой $\Omega$.

3. Алгебраическая интерпретация естественной симплектической структуры на $\mathfrak{N}$. Кратко опишем конструкцию, позволяющую дать альтернативное определение симплектической формы $\Omega$ на многообразии невырожденных нуль-пар. Пусть $G=\mathrm{GL}(n+1, \mathbb{R})$ - полная линейная группа порядка $n+1 ; \mathfrak{g}=\mathfrak{g l}(n+1, \mathbb{R})$ - ее алгебра Ли; $\mathrm{Ad}: G \rightarrow \operatorname{End}(\mathfrak{g})$ - присоединенное представление, $\operatorname{Ad}_{g} \xi=g \xi g^{-1} \forall g \in G, \forall \xi \in \mathfrak{g} ;$ $\langle\cdot, \cdot\rangle$-метрика Киллинга (эта метрика отличается от стандартной метрики Киллинга (см., например, [3, лекция 18, пример 1]) на постоянный множитель) на g:

$$
\langle\xi, \eta\rangle=-\operatorname{tr}(\xi \cdot \eta)+\frac{1}{n+1} \operatorname{tr} \xi \cdot \operatorname{tr} \eta \quad \forall \xi, \eta \in \mathfrak{g} .
$$


Рассмотрим групу $H=\mathrm{GL}(1, \mathbb{R}) \times \mathrm{GL}(n, \mathbb{R})$, стандартно вложенную в $G$. Пусть $f=\operatorname{Ad}\left(Z_{0}\right): g \mapsto \operatorname{Ad}_{g}\left(Z_{0}\right)=g Z_{0} g^{-1}$ - отображение орбиты элемента

$$
Z_{0}=\left(\begin{array}{cccc}
1 & 0 & \ldots & 0 \\
0 & 0 & \ldots & 0 \\
\vdots & \vdots & \ddots & \vdots \\
0 & 0 & \ldots & 0
\end{array}\right) \in \mathfrak{g}
$$

а $M=f(G)$ - соответствующая орбита в $\mathfrak{g}$. Непосредственная проверка показывает, что групша изотропии элемента $Z_{0}$ совпадает с $H$, и так как $f(g H)=f(g) \forall g \in G$, то существует диффеоморфизм $\widehat{f}: G / H \mapsto M$ однородных пространств $G / H=\mathfrak{N}$ и $M$. Действительно, пусть $Z=f(g) \in \mathfrak{g}$-текущая точка орбиты $M$, а $x=(a ; \xi)=\left(a^{0}: a^{1}: \cdots\right.$ : $\left.a^{n} ; \xi_{0}: \xi_{1}: \cdots: \xi_{n}\right) \in \mathfrak{N}$ - текущая точка многообразия невырожденных нуль-пар. Тогда, как легко видеть, диффеоморфизм $\widehat{f}$ задается формулой

$$
\widehat{f}:\left(a^{0}: a^{1}: \cdots: a^{n} ; \xi_{0}: \xi_{1}: \ldots: \xi_{n}\right) \mapsto \frac{1}{\xi(a)}\left(\begin{array}{cccc}
a^{0} \xi_{0} & a^{0} \xi_{1} & \ldots & a^{0} \xi_{n} \\
a^{1} \xi_{0} & a^{1} \xi_{1} & \ldots & a^{1} \xi_{n} \\
\vdots & \vdots & \ddots & \vdots \\
a^{n} \xi_{0} & a^{n} \xi_{1} & \ldots & a^{n} \xi_{n}
\end{array}\right) .
$$

Интерпретируя $a$ как столбец высоты $n+1$, а $\xi$ как строку длины $n+1$, последнюю формулу запишем в виде

$$
\widehat{f}:(p(a) ; p(\xi)) \mapsto \frac{1}{\xi \cdot a} a \cdot \xi=\frac{1}{\xi(a)} a \cdot \xi,
$$

где '.' - матричное умножение.

Каноническая симплектическая форма $F$ на $M$ (форма Кириллова-Костана-Сурьо на орбите присоединенного представления) определяется так (см., например, [4, формула (8.65)]):

$$
F_{Z}(X, Y)=\left\langle Z,\left[\operatorname{ad}_{Z} X, \operatorname{ad}_{Z} Y\right]\right\rangle \quad \forall X, Y \in \operatorname{Imad}_{Z} \cong T_{Z} M .
$$

Найдем форму $\widehat{f}^{*} F$, увлеченную с $M$ на $\mathfrak{N}$. Прежде всего найдем касательные векторы $\operatorname{ad}_{Z} X$ и $\operatorname{ad}_{Z} Y$. Имеем

$$
\begin{aligned}
& \operatorname{ad}_{Z} X=[Z, X]=\frac{1}{\xi \cdot a}(a \cdot \xi \cdot X-X \cdot a \cdot \xi)=\frac{1}{\xi(a)}(a \cdot \eta-b \cdot \xi), \\
& \operatorname{ad}_{Z} Y=[Z, Y]=\frac{1}{\xi \cdot a}(a \cdot \xi \cdot Y-Y \cdot a \cdot \xi)=\frac{1}{\xi(a)}(a \cdot \zeta-c \cdot \xi),
\end{aligned}
$$

где $\eta=\xi \cdot X, b=X \cdot a, \zeta=\xi \cdot Y, c=Y \cdot a$.

Далее находим

$$
\begin{aligned}
{\left[\operatorname{ad}_{Z} X, \operatorname{ad}_{Z} Y\right]=} & \frac{1}{\xi(a) \xi(a)}[(a \cdot \eta-b \cdot \xi),(a \cdot \zeta-c \cdot \xi)] \\
= & \frac{1}{\xi(a) \xi(a)}(a \cdot \eta \cdot a \cdot \zeta-a \cdot \eta \cdot c \cdot \xi-b \cdot \xi \cdot a \cdot \zeta+b \cdot \xi \cdot c \cdot \xi \\
& -a \cdot \zeta \cdot a \cdot \eta+a \cdot \zeta \cdot b \cdot \xi+c \cdot \xi \cdot a \cdot \eta-c \cdot \xi \cdot b \cdot \xi) \\
= & \frac{1}{\xi(a) \xi(a)}(\eta(a) a \cdot \zeta-\eta(c) a \cdot \xi-\xi(a) b \cdot \zeta+\xi(c) b \cdot \xi \\
& -\zeta(a) a \cdot \eta+\zeta(b) a \cdot \xi+\xi(a) c \cdot \eta-\xi(b) c \cdot \xi) .
\end{aligned}
$$


Следовательно,

$$
\begin{aligned}
\left.\left\langle Z, \operatorname{ad}_{Z} X, \operatorname{ad}_{Z} Y\right]\right\rangle= & -\operatorname{tr}\left(Z \cdot\left[\operatorname{ad}_{Z} X, \operatorname{ad}_{Z} Y\right]\right)+\frac{1}{n+1} \operatorname{tr} Z \operatorname{tr}\left(\left[\operatorname{ad}_{Z} X, \operatorname{ad}_{Z} Y\right]\right) \\
= & -\frac{1}{\xi(a) \xi(a) \xi(a)} \operatorname{tr}(a \cdot \xi \cdot(\eta(a) a \cdot \zeta-\eta(c) a \cdot \xi-\xi(a) b \cdot \zeta+\xi(c) b \cdot \xi \\
& -\zeta(a) a \cdot \eta+\zeta(b) a \cdot \xi+\xi(a) c \cdot \eta-\xi(b) c \cdot \xi)) \\
& +\frac{1}{(n+1) \xi(a) \xi(a) \xi(a)} \operatorname{tr}(a \cdot \xi) \operatorname{tr}(\eta(a) a \cdot \zeta-\eta(c) a \cdot \xi-\xi(a) b \cdot \zeta \\
& +\xi(c) b \cdot \xi-\zeta(a) a \cdot \eta+\zeta(b) a \cdot \xi+\xi(a) c \cdot \eta-\xi(b) c \cdot \xi) \\
= & \frac{1}{\xi(a) \xi(a)}(\xi(a) \eta(c)-\xi(c) \eta(a)-\xi(a) \zeta(b)+\xi(b) \zeta(a)) \\
= & \frac{1}{\xi(a) \xi(a)}\left(\begin{array}{ll}
\xi(a) & \xi(c) \\
\eta(a) & \eta(c)
\end{array}|-| \begin{array}{ll}
\xi(a) & \xi(b) \\
\zeta(a) & \zeta(b)
\end{array} \mid\right)
\end{aligned}
$$

Итак,

$$
\widehat{f}^{*} F_{Z}(X, Y)=\frac{1}{\xi(a) \xi(a)}\left(\begin{array}{cc}
\xi(a) & \xi(c) \\
\eta(a) & \eta(c)
\end{array}|-| \begin{array}{cc}
\xi(a) & \xi(b) \\
\zeta(a) & \zeta(b)
\end{array} \mid\right)
$$

где

$$
Z=\frac{1}{\xi(a)} a \cdot \xi, \quad \eta=\xi \cdot X, \quad b=X \cdot a, \quad \zeta=\xi \cdot Y, \quad c=Y \cdot a .
$$

Преобразуем последнюю формулу:

$$
\begin{aligned}
\widehat{f}^{*} F_{Z}(X, Y)= & \frac{1}{\left(\xi_{w} a^{w}\right)^{2}}\left(\left|\begin{array}{cc}
\xi_{w} a^{w} & \xi_{u} d x^{u}(c) \\
d x_{u}(\eta) a^{u} & d x_{u}(\eta) d x^{u}(c)
\end{array}\right|-\left|\begin{array}{cc}
\xi_{w} a^{w} & \xi_{u} d x^{u}(b) \\
d x_{u}(\zeta) a^{u} & d x_{u}(\zeta) d x^{u}(b)
\end{array}\right|\right) \\
= & \frac{1}{\left(\xi_{w} a^{w}\right)^{2}}\left(\xi_{w} a^{w} d x_{u}(\eta) d x^{u}(c)-\xi_{u} d x^{u}(c) d x_{v}(\eta) a^{v}\right. \\
& \left.-\xi_{u} a^{w} d x_{u}(\zeta) d x^{u}(b)+\xi_{u} d x^{u}(b) d x_{v}(\zeta) a^{v}\right) \\
= & \frac{1}{\left(\xi_{w} a^{w}\right)^{2}}\left(\xi_{w} a^{w} \delta_{u}^{v} d x_{v}(\eta) d x^{u}(c)-\xi_{u} d x^{u}(c) d x_{v}(\eta) a^{v}\right. \\
& \left.-\xi_{w} a^{w} \delta_{u}^{v} d x_{v}(\zeta) d x^{u}(b)+\xi_{u} d x^{u}(b) d x_{v}(\zeta) a^{v}\right) \\
= & \frac{1}{\left(\xi_{w} a^{w}\right)^{2}}\left(\xi_{w} a^{w} \delta_{u}^{v}-\xi_{u} a^{v}\right)\left(d x_{u}(\eta) d x^{v}(c)-d x_{u}(\zeta) d x^{u}(b)\right) .
\end{aligned}
$$

Следовательно,

$$
\widehat{f}^{*} F=\frac{1}{\left(x_{w} x^{w}\right)^{2}}\left(\left(x_{w} x^{w}\right) \delta_{u}^{v}-x_{u} x^{v}\right) d x_{u} \wedge d x^{v}
$$

и мы приходим к формуле (3). Производя далее в обратном порядке вычисления из п. 1 в доказательстве предложения 1 , найдем, что ограничения формы $\widehat{f}^{*} F$ на области канонического атласа имеют вид (1). Тем самьм доказано, что симплектическая форма $\Omega$, описанная ранее в терминах канонического атласа, является образом формы Кириллова-Костана-Сурьо при диффеоморфизме отождествления многообразия невырожсденных нуль-пар $\mathfrak{N}$ с орбитой $M$ присоединенного представления груп$n$ bl $\mathrm{GL}(n+1, \mathbb{R})$. Такой подход дает альтернативное доказательство предложения 1 и мотивирует появление явных формул (1) для симплектической структуры $\Omega$. 
ЗАмечАниЕ 3. Вышесказанное объясняет использование термина "естественная" для симлектической структуры $\Omega$. Можно показать, что именно эта структура, но иначе определенная (в терминах форм Маурера-Картана), расмматривалась в работе [2]. Для наших целей наиболее оптимальньм является определение формы $\Omega$ формулой (1) в терминах канонического атласа на $\mathfrak{N}$. Поэтому, а также для единообразия и полноты изложения, в разделе 2 было дано подробное доказательство предложения 1 в терминах канонического атласа.

4. Симплектоморфность многообразия невырожденных нуль-пар кокасателному расслоению проективного пространства. Пусть $P\left(W^{*}\right)_{A}$ - множество всех гиперплоскостей в $P(W)$, не проходящих через точку $A \in P(W)$. Зафиксируем вектор $a \in \mathrm{p}^{-1}(A)$ и рассмотрим отображение

$$
\rho_{a}: P\left(W^{*}\right)_{A} \times P\left(W^{*}\right)_{A} \rightarrow T_{A}^{*} P(W),
$$

определяемое соотношением

$$
\rho_{a}(\mathrm{p}(\xi), \mathrm{p}(\eta))=\left(\mathrm{p}_{a}^{*}\right)^{-1}\left(\frac{1}{\eta(a)} \eta-\frac{1}{\xi(a)} \xi\right) .
$$

Напомним, что $\left(\mathrm{p}_{a}^{*}\right)^{-1}: \operatorname{Ann}\langle a\rangle \rightarrow T_{A}^{*} P(W)$ - изоморфизм, описанньй в разделе 1 . Так как

$$
\begin{aligned}
\rho_{\lambda a}(\mathrm{p}(\xi), \mathrm{p}(\eta)) & =\left(\mathrm{p}_{\lambda a}^{*}\right)^{-1}\left(\frac{1}{\eta(\lambda a)} \eta-\frac{1}{\xi(\lambda a)} \xi\right)=\lambda\left(\mathrm{p}_{a}^{*}\right)^{-1}\left(\frac{1}{\lambda \eta(a)} \eta-\frac{1}{\lambda \xi(a)} \xi\right) \\
& =\rho_{a}(\mathrm{p}(\xi), \mathrm{p}(\eta))
\end{aligned}
$$

то $\rho_{\lambda a}=\rho_{a}$. Следовательно, отображение $\rho_{a}$ не зависит от выбора вектора $a$, порождающего точку $A$. Это отображение будем обозначать через $\rho_{A}$.

Лемма 1. Отображсение $\rho_{A}$ определяет на $P\left(W^{*}\right)_{A}$ структуру аффинного пространства, ассоциированным векторным пространством которого является пространство $T_{A}^{*} P(W)$.

ДоКАЗАТЕЛЬСтво. Проверим аксиомы Вейля аффинного пространства.

1. Пусть $\theta$ - произвольньй элемент из $T_{A}^{*} P(W)$, а $\mathrm{p}(\xi)$ - произвольная гиперплоскость из $P\left(W^{*}\right)_{A}$. Тогда существует единственная гиперплоскость $\mathrm{p}(\eta)$ из $P\left(W^{*}\right)_{A}$, удовлетворяющая условию $\rho_{A}(\mathrm{p}(\xi), \mathrm{p}(\eta))=\theta$. Действительно, пусть

$$
\eta=\mathrm{p}_{a}^{*}(\theta)+\frac{1}{\xi(a)} \xi .
$$

Тогда из формулы (9) и свойства $\mathrm{p}_{a}^{*}(\theta) \in \operatorname{Ann}\langle a\rangle$ получим

$$
\begin{aligned}
\rho_{A}(\mathrm{p}(\xi), \mathrm{p}(\eta)) & =\left(\mathrm{p}_{a}^{*}\right)^{-1}\left(\frac{\eta}{\eta(a)}-\frac{\xi}{\xi(a)}\right)=\left(\mathrm{p}_{a}^{*}\right)^{-1}\left(\frac{\mathrm{p}_{a}^{*}(\theta)+\frac{1}{\xi(a)} \xi}{\mathrm{p}_{a}^{*}(\theta)(a)+\frac{1}{\xi(a)} \xi(a)}-\frac{1}{\xi(a)} \xi\right) \\
& =\left(\mathrm{p}_{a}^{*}\right)^{-1}\left(\mathrm{p}_{a}^{*}\right)(\theta)=\theta .
\end{aligned}
$$


Kроме того, в силу свойства $\mathrm{p}_{a}^{*}(\theta) \in \operatorname{Ann}\langle a\rangle$ найдем, что $\eta(a)=1 \neq 0$. Следовательно, $\mathrm{p}(\eta) \in P\left(W^{*}\right)_{A}$. Пусть теперь $\mathrm{p}\left(\eta^{\prime}\right)$ - другая точка из $P\left(W^{*}\right)_{A}$, удовлетворяющая условию $\rho_{A}\left(\mathrm{p}(\xi), \mathrm{p}\left(\eta^{\prime}\right)\right)=\theta$. Тогда

$$
\frac{\eta(x)}{\eta(a)}-\frac{\xi(x)}{\xi(a)}=\frac{\eta^{\prime}(x)}{\eta^{\prime}(a)}-\frac{\xi(x)}{\xi(a)} \quad \forall x \in W .
$$

Следовательно,

$$
\eta^{\prime}=\frac{\eta^{\prime}(a)}{\eta(a)} \eta \quad \text { и } \quad \mathrm{p}\left(\eta^{\prime}\right)=\mathrm{p}(\eta) .
$$

2. Проверим аксиому треугольника. Так как

$$
\left(\frac{\eta}{\eta(a)}-\frac{\xi}{\xi(a)}\right)+\left(\frac{\zeta}{\zeta(a)}-\frac{\eta}{\eta(a)}\right)=\frac{\zeta}{\zeta(a)}-\frac{\xi}{\xi(a)}
$$

в силу линейности отображения $\left(\mathrm{p}_{a}^{*}\right)^{-1}$ получим

$$
\rho_{A}(\mathrm{p}(\xi), \mathrm{p}(\eta))+\rho_{A}(\mathrm{p}(\eta), \mathrm{p}(\zeta))=\rho_{A}(\mathrm{p}(\xi), \mathrm{p}(\zeta))
$$

СЛЕДСТВИЕ. Пусть $\mathfrak{N}$ - многообразие невырожденных нуль-пар проективного пространства $P(W)$, а $\mathfrak{N}_{A}-$ подмногообразие нуль-пар $(A, \Xi)$ с фиксированной точкой $A$. Тогда подмногообразие $\mathfrak{N}_{A}$ несет структуру аффинного пространства, ассочиированное векторное пространство которого канонически изоморфно кокасательному пространству $T_{A}^{*} P(W) \kappa$ проективному пространству в точке $A$.

Обозначим через $\pi_{1}: \mathfrak{N} \equiv\left(P(W) \times P\left(W^{*}\right)\right) \backslash \mathfrak{M}_{0} \rightarrow P(W)$ проекцию на первьй сомножитель. Ясно, что $\pi_{1}$ - гладкая регулярная сюръекция. Пусть $\mathbb{A}^{n}$ - арифметическое аффинное пространство и $\operatorname{Aff}(n, \mathbb{R})$ - группа аффинных преобразований пространства $\mathbb{A}^{n}$, рассматриваемая как полупрямое произведение групп $\mathrm{GL}(n, \mathbb{R})$ и $\mathbb{R}^{n}$. Напомним, что умножение в $\operatorname{Aff}(n, \mathbb{R})$ определяется как

$$
(A ; x) \cdot(B ; y)=(A B ; A y+x) \quad \forall A, B \in \mathrm{GL}(n, \mathbb{R}), \quad \forall x, y \in \mathbb{R}^{n}
$$

Лемма 2. Проекиия $\pi_{1}: \mathfrak{N} \rightarrow P(W)$ определяет локально тривиальное расслоение (в смысле [5, гл. II, п. 1, определение 1]) с тотальным пространством $\mathfrak{N}$, базой $P(W)$, проекчией $\pi_{1}$, типичным слоем $\mathbb{A}^{n}$ и структурной әруппой $\operatorname{Aff}(n, \mathbb{R})$. Атлас этого расслоения однозначно определяется аффинным атласом кокасательного расслоения $T^{*} P(W)$ и аффинными отображсениями $\rho_{A}$ для всех $A \in P(W)$. При этом слой $\mathfrak{N}_{A}=\pi_{1}^{-1}(A)$ над точкой $A$ несет каноническую структуру аффинного пространства $\mathbb{A}^{n}$, ассочиированным векторным пространством которого является кокасательное пространство $T_{A}^{*} P(W)$ к проективному пространству в точке $A$. 
ДокаЗАтельСтво. Пусть $\mathscr{A}=\left\{\left(A_{p}, f_{p}\right) \mid p=0,1, \ldots, n\right\}$ - аффинньй атлас на $P(W)$. Каноническая структура векторного расслоения определяется на $T^{*} P(W)$ при помощи атласа $\widehat{\mathscr{U}}=\left\{\left(\widehat{U}_{p}, \widehat{F}_{p}\right) \mid p=0,1, \ldots, n\right\}$, где $\widehat{U}_{p}=\pi^{-1}\left(A_{p}\right), \pi: T^{*} P(W) \rightarrow P(W)$ - каноническая проекция, а $\widehat{F}_{p}: \widehat{U}_{p} \rightarrow A_{p} \times \mathbb{R}^{n}$ - тривиализирующие диффеоморфизмы, определяемые соотношениями

$$
\widehat{F}_{p}:\left(A, \omega_{A}\right) \mapsto\left(A,\left(f_{p}^{-1}\right)^{*} \omega_{A}\right) .
$$

Рассмотрим теперь семейство открытых множеств $\left\{U_{p}\right\}_{p=0,1, \ldots, n}$ на $\mathfrak{N}$, где $U_{p}=$ $\pi_{1}^{-1}\left(A_{p}\right)$, а $\pi_{1}: \mathfrak{N} \equiv P(W) \times P\left(W^{*}\right) \backslash \mathfrak{M}_{0} \rightarrow P(W)$ - проекция на первый сомножитель. Ясно, что $\left\{U_{p}\right\}_{p=0,1, \ldots, n}$ - открытое покрытие многообразия $\mathfrak{N}$. Для каждого $U_{p}$ рассмотрим отображение $F_{p}: U_{p} \rightarrow A_{p} \times \mathbb{A}^{n}$, определяемое соотношениями

$$
F_{p}:(A, \Xi) \mapsto\left(A,\left(f_{p}^{-1}\right)^{*} \circ \rho_{A}\left(E^{p}, \Xi\right)\right),
$$

где $E^{p}=\mathrm{p}\left(e^{p}\right)$, a $e^{p}$ - базисньй ковектор с номером $p$. Обратим внимание на то, что

$$
F_{p}(u)=\widehat{F}_{p} \circ \rho_{\pi_{1}(u)}\left(\pi_{1}(u), E^{p}\right) \quad \forall u \in U_{p}=\pi_{1}^{-1}\left(A_{p}\right),
$$

т.е. отображение $F_{p}$ определяется при помощи диффеоморфизма $\widehat{F}_{p}$ и аффинных отображений $\rho_{A}$, где $A \in A_{p} \subset P(W)$. Несложно проверить, что $F_{p}$ - диффеоморфизм для каждого $p$. Следовательно, $\mathscr{U}=\left\{\left(U_{p}, F_{p}\right) \mid p=0,1, \ldots, n\right\}$ - допустимый атлас на $\mathfrak{N}$.

Докажем, что атлас $\mathscr{U}$ определяет на $\mathfrak{N}$ структуру искомого расслоения. Если $A \in$ $A_{p} \cap A_{q}$, то

$$
F_{q}:(A, \Xi) \mapsto\left(A,\left(f_{q}^{-1}\right)^{*} \circ \rho_{A}\left(E^{q}, \Xi\right)\right) .
$$

Функция перехода

$$
F_{q p} \equiv F_{q} \circ F_{p}^{-1}:\left(A_{p} \cap A_{q}\right) \times \mathbb{A}^{n} \rightarrow\left(A_{p} \cap A_{q}\right) \times \mathbb{A}^{n}
$$

определяется так:

$$
F_{q p}:\left(A,\left(f_{p}^{-1}\right)^{*} \circ \rho_{A}\left(E^{p}, \Xi\right)\right) \mapsto\left(A,\left(f_{q}^{-1}\right)^{*} \circ \rho_{A}\left(E^{q}, \Xi\right)\right) .
$$

С другой стороны, пусть $x=\left(f_{p}^{-1}\right)^{*} \circ \rho_{A}\left(E^{p}, \Xi\right) \in \mathbb{A}^{n}$. Тогда

$$
\begin{aligned}
\left(f_{q}^{-1}\right)^{*} \circ \rho_{A}\left(E^{q}, \Xi\right) & =\left(f_{q}^{-1}\right)^{*}\left(\rho_{A}\left(E^{q}, E^{p}\right)+\rho_{A}\left(E^{p}, \Xi\right)\right) \\
& =\left(f_{q}^{-1}\right)^{*} \circ \rho_{A}\left(E^{q}, E^{p}\right)+\left(f_{q}^{-1}\right)^{*} \circ \rho_{A}\left(E^{p}, \Xi\right) \\
& =C_{q p}+\left(f_{q}^{-1}\right)^{*} \circ\left(f_{p}\right)^{*} \circ\left(f_{p}^{-1}\right)^{*} \circ \rho_{A}\left(E^{p}, \Xi\right) \\
& =C_{q p}+\left(f_{p} \circ f_{q}^{-1}\right)^{*} \circ\left(f_{p}^{-1}\right)^{*} \circ \rho_{A}\left(E^{p}, \Xi\right) \\
& =C_{q p}+\left(f_{p} \circ f_{q}^{-1}\right)^{*}\left(\left(f_{p}^{-1}\right)^{*} \circ \rho_{A}\left(E^{p}, \Xi\right)\right)=C_{q p}+\left(f_{p} \circ f_{q}^{-1}\right)^{*}(x),
\end{aligned}
$$

где $C_{q p}=\left(f_{q}^{-1}\right)^{*} \circ \rho_{A}\left(E^{q}, E^{p}\right)$ - постоянный вектор в $\mathbb{R}^{n}, \mathrm{a}\left(f_{p} \circ f_{q}^{-1}\right)^{*}$ - элемент группы $\mathrm{GL}(n, \mathbb{R})$ для каждой точки $A \in A_{p} \cap A_{q}$. Итак,

$$
F_{q p}:(A, x) \mapsto\left(A, C_{q p}+\left(f_{p} \circ f_{q}^{-1}\right)^{*}(x)\right),
$$


т.е. имеем отображение $h_{q p}: A_{p} \cap A_{q} \rightarrow \operatorname{Aff}(n, \mathbb{R})$ из $A_{p} \cap A_{q}$ в группу Ли $\operatorname{Aff}(n, \mathbb{R})$ аффинных преобразований пространства $\mathbb{A}^{n}$, определяемое по закону

$$
h_{q p}(A)=\left(\left(f_{p} \circ f_{q}^{-1}\right)_{A}^{*},\left(f_{q}^{-1}\right)^{*} \circ \rho_{A}\left(E^{q}, E^{p}\right)\right) \in \operatorname{Aff}(n, \mathbb{R}) .
$$

Аффинное преобразование $h_{q p}(A)$ соответствует переходу от карты $\left(A_{p}, f_{p}\right)$ к карте $\left(A_{q}, f_{q}\right)$ атласа $\mathscr{A}$. Пусть теперь $A \in A_{p} \cap A_{q} \cap A_{r}$. Тогда

$$
h_{r q}(A)=\left(\left(f_{q} \circ f_{r}^{-1}\right)_{A}^{*} ;\left(f_{r}^{-1}\right)^{*} \circ \rho_{A}\left(E^{r}, E^{q}\right)\right) \in \operatorname{Aff}(n, \mathbb{R}) \text {. }
$$

Следовательно,

$$
\begin{aligned}
h_{r q} & (A) \cdot h_{q p}(A)=\left(\left(f_{q} f_{r}^{-1}\right)_{A}^{*} ;\left(f_{r}^{-1}\right)_{A}^{*} \rho_{A}\left(E^{r}, E^{q}\right)\right) \cdot\left(\left(f_{p} f_{q}^{-1}\right)_{A}^{*} ;\left(f_{q}^{-1}\right)_{A}^{*} \rho_{A}\left(E^{q}, E^{p}\right)\right) \\
& =\left(\left(f_{q} f_{r}^{-1}\right)_{A}^{*}\left(f_{p} f_{q}^{-1}\right)_{A}^{*} ;\left(f_{q} f_{r}^{-1}\right)_{A}^{*}\left(f_{q}^{-1}\right)_{A}^{*} \rho_{A}\left(E^{q}, E^{p}\right)+\left(f_{r}^{-1}\right)_{A}^{*} \rho_{A}\left(E^{r}, E^{q}\right)\right) \\
& =\left(\left(f_{p} f_{r}^{-1}\right)_{A}^{*} ;\left(f_{r}^{-1}\right)_{A}^{*}\left(\rho_{A}\left(E^{q}, E^{p}\right)+\rho_{A}\left(E^{r}, E^{q}\right)\right)\right) \\
& =\left(\left(f_{p} f_{r}^{-1}\right)_{A}^{*} ;\left(f_{r}^{-1}\right)_{A}^{*}\left(-\rho_{A}\left(E^{p}, E^{q}\right)-\rho_{A}\left(E^{q}, E^{r}\right)\right)\right) \\
& =\left(\left(f_{p} f_{r}^{-1}\right)_{A}^{*} ;\left(f_{r}^{-1}\right)_{A}^{*}\left(-\rho_{A}\left(E^{p}, E^{r}\right)\right)\right) \\
& =\left(\left(f_{p} f_{r}^{-1}\right)_{A}^{*} ;\left(f_{r}^{-1}\right)_{A}^{*} \rho_{A}\left(E^{r}, E^{p}\right)\right)=h_{r p}(A) .
\end{aligned}
$$

Таким образом, $h_{r p}(A)=h_{r q}(A) \cdot h_{q p}(A) \forall A \in A_{p} \cap A_{q} \cap A_{r}$.

Итак, имеем локально тривиальное расслоение с тотальным пространством $\mathfrak{N}$, базой $P(W)$, проекцией $\pi_{1}$, типичным слоем $\mathbb{A}^{n}$, структурной группой $\operatorname{Aff}(n, \mathbb{R})$ и тривиализирующим атласом $\mathscr{U}$. Слой $\mathfrak{N}_{A}=\pi_{1}^{-1}(A)$ над точкой $A$ несет каноническую структуру аффинного пространства $\mathbb{A}^{n}$, ассоциированньм векторным пространством которого является кокасательное пространство $T_{A}^{*} P(W)$ к проективному пространству в точке $A$.

Лемма 3. Расслоение $\pi_{1}: \mathfrak{N} \rightarrow P(W)$ допускает сечение.

ДокАЗАтЕльСтво. Обозначим через $g=(\cdot, \cdot)$ стандартное скалярное произведение в $W=\mathbb{R}^{n+1}$. Операция опускания индекса при помоши метрики $g$ определяет изоморфизм $i_{g}$ векторных пространств $W$ и $W^{*}$. Ковектор $i_{g}(a): x \mapsto(a, x) \forall x \in W$, соответствующий вектору $а$ при этом изоморфизме, будем обозначать через $\widehat{a}$. Изоморфизм $i_{g}$ индуцирует отображение $s$ из $P(W)$ в $P\left(W^{*}\right)$ по закону $s: \mathrm{p}(a) \mapsto \mathrm{p}(\widehat{a})$. Так как $\widehat{a}(a)=(a, a) \neq 0$ для любого $a \neq 0$, то $s(A) \in \mathfrak{N}_{A} \forall A \in P(W)$. Кроме того, очевидно, что $\pi_{1} \circ s=\operatorname{id}_{P(W)}$. Следовательно, $s$ - сечение.

Лемма 4. Многообразие невырожсенных нуль-пар $\mathfrak{N}$ диффеоморфно кокасательному расслоению $T^{*} P(W)$. Диффеоморфизм устанавливается при помощи отображсния

$$
\sigma: \mathfrak{N} \ni(A, \Xi) \mapsto\left(A, \rho_{A}(s(A), \Xi)\right) \in T_{A}^{*} P(W) .
$$

Более подробно, невырохсенной нуль-паре $x=(A, \Xi)=(\mathrm{p}(a), \mathrm{p}(\xi))$ соответствует линейная форма $\sigma(x)=\omega \in T_{A}^{*} P(W)$, определяемая соотношением

$$
\omega_{A}=\left(\mathrm{p}_{a}^{*}\right)^{-1}\left(\frac{\xi}{\xi(a)}-\frac{\widehat{a}}{\widehat{a}(a)}\right) \quad \forall a \in \mathrm{p}^{-1}(A) .
$$


ДокАЗАтЕльство. 1. Очевидно, что отображение $\sigma$ биективно, так как оно обратимо. Отображение $\sigma^{-1}$ определяется как

$$
\sigma^{-1}: T_{A}^{*} P(W) \ni\left(A, \omega_{A}\right) \mapsto(A, \mathrm{p}(\xi)) \in \mathfrak{N},
$$

где

$$
\xi=\mathrm{p}_{a}^{*} \omega+\frac{\widehat{a}}{\widehat{a}(a)} \quad \forall a \in \mathrm{p}^{-1}(A) .
$$

2. Докажем, что $\sigma$ и $\sigma^{-1}-$ гладкие отображения.

Пусть $y^{i_{p}}$ и $y_{j_{p}}-$ локальные координаты на $\mathfrak{N}$ в области $\left(A_{p} \times A^{p}\right) \cap \mathfrak{N}$, а $z_{i_{p}}$ и $z^{i_{p}}$ локальные координаты на $T^{*} P(W)$ в области $\widehat{U}_{p}$. Тогда из $(10)$ находим, что локальная запись отображения $\sigma$ имеет вид

$$
\widehat{F}_{p} \circ \sigma \circ\left(f_{p} \times f^{p}\right)^{-1}:\left\{\begin{array}{l}
z^{i_{p}}=y^{i_{p}} \\
z_{i_{p}}=\frac{y_{i_{p}}}{1+y_{j_{p}} y^{j_{p}}}-\frac{y^{i_{p}}}{1+\sum y^{j_{p}} y^{j_{p}}} .
\end{array}\right.
$$

Ясно, что $\sigma$ - гладкое отображение. Дифференцируя, найдем

$$
\begin{aligned}
\left(\widehat{F}_{p} \circ \sigma \circ\left(f_{p} \times f^{p}\right)^{-1}\right)^{*} d z^{i_{p}}= & d y^{i_{p}}, \\
\left(\widehat{F}_{p} \circ \sigma \circ\left(f_{p} \times f^{p}\right)^{-1}\right)^{*} d z_{i_{p}}= & \frac{\left(1+y_{j_{p}} y^{j_{p}}\right) d y_{i_{p}}-y_{i_{p}}\left(d y_{j_{p}} y^{j_{p}}+y_{j_{p}} d y^{j_{p}}\right)}{\left(1+y_{j_{p}} y^{j_{p}}\right)^{2}} \\
& -\frac{\left(1+\sum y^{j_{p}} y^{j_{p}}\right) d y^{i_{p}}-2 y^{i_{p}} \sum y^{j_{p}} d y^{j_{p}}}{\left(1+\sum y^{j_{p}} y^{j_{p}}\right)^{2}} .
\end{aligned}
$$

Из (12), (13) следует, что матрища Якоби отображения $\sigma$ имеет вид

$$
\mathbf{J}=\left(\begin{array}{cc}
\left(\delta_{i_{p}}^{j_{p}}\right) & 0 \\
\left(\frac{2 y^{i_{p}} y^{j_{p}}-\mu \delta^{i_{p} j_{p}}}{\mu^{2}}-\frac{y_{i_{p}} y_{j_{p}}}{\lambda^{2}}\right) & \left(\frac{\lambda \delta_{i_{p}}^{j_{p}}-y_{i_{p}} y^{j_{p}}}{\lambda^{2}}\right)
\end{array}\right),
$$

где $\lambda=1+y_{j_{p}} y^{j_{p}}$ и $\mu=1+\sum y^{j_{p}} y^{j_{p}}$. Так как

$$
\operatorname{det} \mathbf{J}=\operatorname{det}\left(\frac{\lambda \delta_{i_{p}}^{j_{p}}-y_{i_{p}} y^{j_{p}}}{\lambda^{2}}\right)=\frac{1}{\lambda^{2 n}}\left(\lambda^{n}-\lambda^{n-1} y_{j_{p}} y^{j_{p}}\right)=\frac{1}{\lambda^{n+1}} \neq 0
$$

то отображение $\sigma$ являет регулярным в области $\left(A_{p} \times A^{p}\right) \cap \mathfrak{N}$. Отображение $\sigma^{-1}$ определяется в области $\sigma\left(\left(A_{p} \times A^{p}\right) \cap \mathfrak{N}\right) \subset \widehat{U}_{p}$ формулой

$$
\left(f_{p} \times f^{p}\right) \circ \sigma^{-1} \circ \widehat{F}_{p}^{-1}:\left\{\begin{array}{l}
y^{i_{p}}=z^{i_{p}} \\
y_{i_{p}}=\frac{\left(1+\sum z^{k_{p}} z^{k_{p}}\right) z_{i_{p}}+z^{i_{p}}}{1-z_{j_{p}} z^{j_{p}}\left(1+\sum z^{k_{p}} z^{k_{p}}\right)} .
\end{array}\right.
$$

Так как для любой точки из $\sigma\left(\left(A_{p} \times A^{p}\right) \cap \mathfrak{N}\right)$ имеет место формула

$$
1-z_{j_{p}} z^{j_{p}}\left(1+\sum z^{k_{p}} z^{k_{p}}\right)=\frac{1+\sum y^{k_{p}} y^{k_{p}}}{1+y_{j_{p}} y^{j_{p}}} \neq 0
$$

то $\sigma^{-1}-$ гладкое отображение.

Итак, $\sigma$ и $\sigma^{-1}$ - гладкие биекции. Следовательно, $\sigma$ - диффеоморфизм.

Теперь мы можем сформулировать основной результат. 
ТЕОремА. Многообразие невырожденных нуль-пар $\mathfrak{N}$ проективного пространства $P(W)$ симплектоморфно кокасательному расслоению $T^{*} P(W)$ проективному пространству $P(W)$.

ДокАЗАТЕЛЬСтво. Докажем, что диффеоморфизм $\sigma: \mathfrak{N} \rightarrow T^{*} P(W)$ является симплектоморфизмом относительно естественной симплектической структуры на $\mathfrak{N}$ и канонической симплектической структуры на $T^{*} P(W)$.

Пусть $\omega$ - стандартная форма Лиувилля [6] на $T^{*} P(W)$, определяемая равенством

$$
\omega(X)_{\theta}=\theta\left(\pi_{*}(X)\right)_{\pi(\theta)} \quad \forall \theta \in T^{*} P(W), \quad \forall X \in \mathfrak{X}\left(T^{*} P(W)\right) .
$$

Тогда $\Theta=d \omega-$ каноническая симплектическая форма на $T^{*} P(W)$. В локальных координатах $\left\{z^{i_{p}}, z_{j_{p}}\right\}$ на $\widehat{U}_{p} \subset T^{*} P(W)$ форма $\Theta$ имеет вид

$$
\left.\Theta\right|_{\widehat{U}_{p}}=F_{p}^{*} \underset{p}{\Theta}, \quad \text { где } \underset{p}{\Theta}=d z_{i_{p}} \wedge d z^{i_{p}}
$$

Ясно, что $\sigma^{*} \Theta$ - некоторая симплектическая форма на $\mathfrak{N}$, так как $\sigma$ - диффеоморфизм. Используя формулы (12), (13), находим

$$
\begin{aligned}
\left.\sigma^{*} \Theta\right|_{\widehat{U}_{p}} & =\sigma^{*} \circ F_{p}^{*} \underset{p}{\Theta}=\sigma^{*} \circ F_{p}^{*} d z_{i_{p}} \wedge d z^{i_{p}}=\sigma^{*} \circ F_{p}^{*} d z_{i_{p}} \wedge \sigma^{*} \circ F_{p}^{*} d z^{i_{p}} \\
& =\left(f_{p} \times f^{p}\right)^{*}\left(\left(\left(f_{p} \times f^{p}\right)^{-1}\right)^{*} \circ \sigma^{*} \circ F_{p}^{*} d z_{i_{p}} \wedge\left(\left(f_{p} \times f^{p}\right)^{-1}\right)^{*} \circ \sigma^{*} \circ F_{p}^{*} d z^{i_{p}}\right) \\
& =\left(f_{p} \times f^{p}\right)^{*}\left(\frac{1}{\left(1+y_{k_{p}} y^{k_{p}}\right)^{2}}\left(\left(1+y_{k_{p}} y^{k_{p}}\right) \delta_{j_{p}}^{i_{p}}-y^{i_{p}} y_{j_{p}}\right) d y_{i_{p}} \wedge d y^{j_{p}}\right) \\
& =\left(f_{p} \times f^{p}\right)^{*} \underset{p}{\Omega}=\left.\Omega\right|_{\left(A_{p} \times A^{p}\right) \cap \mathfrak{N} .}
\end{aligned}
$$

Итак,

$$
\left.\sigma^{*} \Theta\right|_{\widehat{U}_{p}}=\left.\Omega\right|_{\left(A_{p} \times A^{p}\right) \cap \mathfrak{N}} \quad \forall p \in\{0,1, \ldots, n\} .
$$

Следовательно, $\sigma^{*} \Theta=\Omega$. Теорема доказана.

ЗАмЕчАНИЕ 4. Простейший пример механической системы, фазовым пространством которой является многообразие невырожденных нуль-пар, строится следующим образом. Пусть внутрь сферы помешен стержень, длина которого равна диаметру сферы. Тогда пространство конфигураций стержня параметризуется точками проективной плоскости $P^{2}$, а фазовое пространство отождествляется с кокасателным расслоением $T^{*} P^{2}$, которое в силу доказанной теоремы симплектоморфно четырехмерному многообразию невырожденных нуль-пар на проективной плоскости.

ЗАмЕчАниЕ 5. Пусть теперь

$$
\mathfrak{M}_{0}=\left\{(\langle a\rangle,\langle\xi\rangle) \in P(W) \times P\left(W^{*}\right) \mid \xi(a)=0\right\}=\bigcup_{a \in W \backslash\{0\}}\langle a\rangle \times P(\operatorname{Ann}\langle a\rangle)
$$

- многообразие вырожденных нуль-пар в проективном пространстве $P(W)$, а

$$
P T^{*} P(W)=\bigcup_{A \in P(W)} P\left(T_{A}^{*} P(W)\right)
$$


- проективизачия кокасательного расслоения пространства $P(W)$, т.е. $P T^{*} P(W)$ многообразие всех проективных пространств $P\left(T_{A}^{*} P(W)\right)$, порожденных кокасательными пространствами $T_{A}^{*} P(W)$ к проективному пространству $P(W)$ во всех его точках $A \in P(W)$. Ясно, что

$$
\operatorname{dim} \mathfrak{M}_{0}=\operatorname{dim} P T^{*} P(W)=2 \cdot \operatorname{dim} P(W)-1=2 n-1
$$

Рассмотрим отображение

$$
\varrho=\mathrm{id} \times \mathrm{p} \circ\left(\mathrm{p}_{a}^{*}\right)^{-1}: \mathfrak{M}_{0} \rightarrow P T^{*} P(W),
$$

определяемое соотношением

$$
\varrho:(A, \Xi) \mapsto\left(A, \mathrm{p} \circ\left(\mathrm{p}_{a}^{*}\right)^{-1}(\xi)\right) \quad \forall a \in \mathrm{p}^{-1}(A), \quad \forall \xi \in \mathrm{p}^{-1}(\Xi)
$$

Taк как

$$
\mathrm{p} \circ\left(\mathrm{p}_{\lambda a}^{*}\right)^{-1}(\mu \xi)=\mathrm{p}\left(\left(\mathrm{p}_{\lambda a}^{*}\right)^{-1}(\mu \xi)\right)=\mathrm{p}\left(\lambda \mu\left(\mathrm{p}_{a}^{*}\right)^{-1}(\xi)\right)=\mathrm{p}\left(\left(\mathrm{p}_{a}^{*}\right)^{-1}(\xi)\right)=\mathrm{p} \circ\left(\mathrm{p}_{a}^{*}\right)^{-1}(\xi)
$$

то отображение $\varrho$ не зависит от выбора вектора $a$ и ковектора $\xi$, порождающих нуль-пару $(A, \Xi)$. Ясно, что $\varrho$ - биекция. Обратное отображение имеет вид

$$
\varrho^{-1}:(A, \mathrm{p}(\theta)) \mapsto\left(A, \mathrm{p} \circ \mathrm{p}_{a}^{*}(\theta)\right) \quad \forall a \in \mathrm{p}^{-1}(A), \quad \forall \theta \in T_{A}^{*} P(W) .
$$

Несложно проверить, что $\varrho-$ диффеоморфизм. Таким образом, многообразие невырожденных нуль-пар $\mathfrak{N}$ в $P(W)$ симплектоморфно кокасательному расслоению $T^{*} P(W)$ проективного пространства $P(W)$, а многообразие вырохденных нульпар $\mathfrak{M}_{0}$ диффеоморфно проективизации $P T^{*} P(W)$ кокасательного расслоения пространства $P(W)$.

\section{СПИСОК ЦИТИРОВАННОЙ ЛИТЕРАТУРЫ}

[1] Розенфельд Б. А. Многомерные пространства. М.: Наука, 1966.

[2] Kirichenko V.F. Generalized quasi-Kaehlerian manifolds and axioms of $C R$-submanifolds in generalized Hermitian geometry, II // Geometriae Dedicata. 1994. V. 52. P. 53-85.

[3] Постников M. M. Лекции по геометрии. Семестр V. Группы и алгебры Ли. М.: Наука, 1982.

[4] Бессе А. Многообразия Эйнштейна. Т. 1. М.: Мир, 1990.

[5] Зуланке Р., Винтген П. Дифференциальная геометрия и расслоения. М.: Мир, 1975.

[6] Годбийон К. Дифференциальная геометрия и аналитическая механика. М.: Мир, 1973.

Самарский государственный педагогический университет

E-mail : vkonnov@fa.ru

Поступило 22.03 .2000

Исправленный вариант 26.09.2000 